\title{
La filosofía de la ciencia como disciplina hermenéutica
}

\author{
ULISES MOULINES \\ Instituto de Filosofia, Lógica y Teoría de la Ciencia \\ Universidad de Munich
}

En este ensayo me propongo desarrollar y sustentar la tesis de que la filosolía de la ciencia es esencialmente una parte de las ciencias de la cultura. Esta tesis puede parecer, a primera vista, trivial. En efecto, todo el mundo estará de acuerdo en que la ciencia es una parte importante de la cultura actual; y por lo tanto, la reflexión filosófica sobre la ciencia, al ser una actividad teórica que pretende aplicarse según ciertos criterios de cientificidad a una parte de la cultura, se incluirá ipso facto en esa área del conocimiento que denominamos «ciencias de la cultura» o whumanidades». Sin embargo, como sucede con frecuencia, las aparentes trivialidades conccptuales tienen importantes consecuencias teóricas y prácticas. El presente caso es, según creo, un ejemplo de ello: el incluir la filosofía de la ciencia dentro del marco más amplio de las ciencias de la cultura tiene consecuencias no negligibles (y quizás imprevistas para algunos) tanto sobre nuestra comprensión de lo que es la filosofía de la ciencia como sobre lo que son, o pueden llegar a ser, las ciencias de la cultura. Poner de manifiesto dichas consecuencias es el propósito de las páginas que siguen.

Permítaseme empezar esta discusion con una especie de atrevida definición. Quisiera caracterizar la filosofía de la ciencia como un tipo particular de teorización sobre teorizaciones. Y quisiera asimilar esta caracterización a esta otra: la filosofia de la ciencia es una interpretación de interpretaciones de la realidad; o bien, para ser un poco más precisos, la filosofía de la ciencia consiste en la construcción de marcos interpretativos filosóficos que nos permiten entender esos marcos interpretativos de la realidad que llamamos «teorias científicas». Naturalmente, esta caracterización de la filosofía de la ciencia como teorización sobre teorizaciones 0 , alternativamente, como interpretación de interpretaciones, es aún muy pobre, casi vacía, dado que no he definido o aclarado las nociones de teorización o interpretación que aquí se proponen. Volveré a estas nociones en un momento. Pero antes de ello, quisiera hacer notar que la caracterización propuesta de la filosofía de la ciencia nos da ya una indicación de su naturaleza: es lo que puede llamarse una actividad intelectual de segundo nivel, es decir, una actividad intelectual cuyos objetos de estudio son en sí mismos ya el resultado de actividades intelectuales. La filosofía de la ciencia es un 
fenómeno recursivo. Creo que es una característica interesante de muchas actividades humanas, ya sean intelectuales o no, el que sean recursivas, es decir, que puedan aplicarse subsiguientemente a sus propios resultados. Por ejemplo, no sólo podemos hablar, sino también hablar sobre el habla; no sólo podemos amar, sino también amar el amor, y así sucesivamente. La filosofía en general, y en particular la filosofía de la ciencia, es un caso típico de actividad recursiva. La clase de actividad que se aplica recursivamente en este caso es lo que llamo teorización o, alternativamente, interpretación. Aunque no puedo ofrecer aquí una definición formal de estas dos nociones, trataré de aproximarme sucesivamente a ellas.

La teorización conșiste en la producción de ciertas entidades que llamamos teorías. Diré más sobre el concepto de teoría posteriormente. Ahora sólo quisiera añadir que considero las teorías como marcos conceptuales interpretativos construidos para entender las cosas. En consecuencia, estoy caracterizando la noción de teorización en términos de la noción de interpretación. Así, pues, el concepto de interpretación resulta ser el central.

Ahora debería decir lo que entiendo por interpretación. No pretendo usar este término en un sentido filosófico. Mi idea de interpretación ha ido cuajando a partir de mi confrontación con un dogma generalmente implicito, pero no menos ampliamente difundido, de la filosofía analítica, a saber, lo que podemos denominar el dogma de la dicotomía descriptivonormativo. De acuerdo con esta dicotomía, cualquier concepto, enunciado o sisterma de enunciados que tenga sentido es o bien descriptivo o bien normativo (prescriptivo), y ambas categorias se excluyen mutuamente. Al asumir esta dicotomía como punto de partida indiscutible, muchos filósofos de la ciencia que reflexionan sobre su propia tarea se han preguntado si el estudio filosófico de la ciencia debería ser considerado como un conjunto de descripciones neutrales del contenido de la ciencia o más bien como un conjunto de normas a priori que deben seguir los científicos. Diversas respuestas se han dado a este dilema, unas favoreciendo decididamente el descriptivismo, otras el normativismo y otras, finalmente, abogando por una combinación a partes iguales del punto de vista normativo con el descriptivo. Pero todas estas diferentes respuestas dan por supuesto que todo lo que uno, como filósofo, puede hacer con respecto a la ciencia es describirla o darle normas de procedimiento.

Pues bien, creo que esta dicotomía así formulada es confundente. Las categorías "descriptivo" y "normativo" se pueden aplicar de manera interesante y fructífera a muchos tipos de discurso, pero en otros casos sólo pueden aplicarse de manera forzada e inverosímil. Por ejemplo, puedo describir lo que veo en el cielo en la noche diciendo que hay un enorme número de puntos luminosos en diferentes posiciones y con diversa intensidad luminosa, algunos de los cuales varían de posición de una manera 
regular, otros de manera irregular. Esto es una descripción. Pero cuando digo que algunos de estos puntos luminosos influyen en nuestro destino o bien que se atraen mutuamente según la ley de gravitación, ya no estoy describiendo nada (en ningún sentido interesante y plausible de la palabra "describir»), sino que estoy «interpretando» mis datos. El resultado de una interpretación semejante es una teoría: en este ejemplo, una teoría astrológica o mecánica. En el otro extremo, puedo redactar un código que fije ciertas normas de comportamiento de la gente. Esto es una actividad normativa. Pero también puedo considerar la conducta de la gente como el resultado de ciertas estructuras institucionales, sociales y económicas, en cuyo caso ya no estoy dando normas, sino interpretando su conducta, es decir, construyendo una teoría sobre ella.

La interpretación es una categoría semántica autónoma, que no puede reducirse ni a la descripción ni a la prescripción. Creo que la filosofia analítica del lenguaje debería tratar de dar una elucidación detallada, formal, de esta "tercera» categoria y de las reglas semánticas a las que está sometida. No he visto ningún intento de esta clase en la literatura de la filosofía analítica. Habría que intentar algo en este sentido. Aquí no puedo entrar a discutir en detalle esta difícil cuestión de semántica filosófica, y también quisiera dejar claro que no dispongo de una teoría sistemática al respecto. Hay que dejar la cuestión planteada para futuras generaciones de lógicos y filósofos. A pesar de ello, sí quisiera esbozar unas indicaciones generales que podrían representar un primer paso para aclarar la noción de interpretación. La disciplina filosófica que tradicionalmente se ha ocupado de la interpretación es la hermenéutica. No obstante, la hermenéutica se ha practicado de diversas maneras, ninguna de las cuales me parece enteramente satisfactoria para nuestros propósitos. En su forma más clási$\mathrm{ca}$, que arranca de Aristóteles, la hermenéutica es simplemente la teoría de los signos, o más exactamente, la teoría de la relación entre el signo y lo designado. En esta forma, la hermenéutica coincide con la semántica formal de la lógica moderna, y dentro de ella, la interpretación no es más que una relación formal entre cierto lenguaje y un dominio de objetos. No es éste el sentido en que aquí queremos hablar de interpretación, puesto que no contiene el aspecto activo de reconstrucción conceptual de un dominio por parte de un sujeto. Otro sentido de hermenéutica que se ha manejado en la filosofía contemporánea es el que arranca de Dilthey y tiene una relación directa con las ciencias de la cultura. En él, la interpretación es una actividad intelectual referida exclusivamente a textos $\mathfrak{u}$ otros objetos culturales con el intento de llegar a una comprensión interna y contextualmente relativa de dichos objetos. Esta operación la aplicó Dilthey, como es sabido, a textos históricos y literarios, y Heidegger y Gadamer la han aplicado a textos filosóficos. Esta idea de interpretación es un poco más cercana a la nuestra que la anterior, sobre todo porque subraya el papel activo 
del investigador; pero también difiere en gran medida, porque Dilthey y los hermeneutas contemporáneos se negaron deliberadamente a usar categorías teóricas generales para formular sus interpretaciones. Su hermenéutica es un enfoque particularista, adverso a la abstracción y generalización. Quizás lo más afín a la idea de interpretación que queremos proponer aquí es la operación de las categorías del entendimiento según Kant, que como sabemos es una operación activa del sujeto sobre el objeto, que no le lleva a la mera comprensión o descripción del objeto, sino a su reconstrucción y asimilación a categorías generales a priori. Es una operación como ésta, o parecida a ésta, la que considero característica de las teorías en todos los campos.

Existen muchas clases de teorías interpretativas sobre la realidad. Hay interpretaciones mitológicas, metafísicas, ideológicas. También existen interpretaciones científicas. El grueso de las ciencias avanzadas, ya șean naturales o sociales, consiste en teorias interpretativas en el sentido postulado aquí. Estas teorías interpretativas son objetos culturales producidos por ciertas personas en ciertos contextos sociales y culturales en determinadas épocas. Estos objetos culturales (que llamaremos en lo sucesivo «teorías del primer nivel") son el objeto de estudio teórico de teorias interpretativas del segundo nivel. Estas últimas constituyen lo que denominamos filosofía de la ciencia. En consecuencia, podemos dar una caracterización más aproximada de la filosofía de la ciencia diciendo que ella consiste en la construcción de interpretaciones de segundo nivel que tienen como objetos de estudio las interpretaciones científicas del primer nivel. Dado que estas últimas son objetos culturales, está claro por qué podemos adscribir la filosofia de la ciencia a las ciencias de la cultura o humanidades.

Es importante señalar en este punto que la interpretación filosófica de las teorias científicas que llamamos filosofía de la ciencia no es la única manera posible de teorizar sobre la ciencia como objeto de estudio. En vez de tratar de las teorías científicas como tales, podriamos tratar de las instituciones sociales o de la gente que las produce. En tales casos, emprenderíamos otro tipo de estudio teórico de la ciencia; por ejemplo, la sociología de la ciencia o la psicología de la ciencia. Ciertamente hay muchas conexiones importantes y fructiferas entre la filosolía de la ciencia, por un lado, y la sociología o psicología de la ciencia, por otro. Pero también deberíamos ser plenamente conscientes de que, contrariamente a una opinión ampliamente difundida, la filosofía de la ciencia y los estudios sociales de la ciencia no pueden identificarse. Sean cuales sean las fucrtes conexiones que se puedan establecer entre ambas disciplinas, sus objetos de estudio son esencialmente distintos. Los estudios sociales de la ciencia tratan de entidades localizadas espacio-temporalmente, tales como investigadores e instituciones académicas, mientras que la filosofía de la ciencia trata de teorias, y éstas son entidades abstractas en el sentido de no estar localiza- 
das espacio-temporalmente, al menos no en el mismo sentido en que lo están los organismos o las entidades sociales. El filósofo de la ciencia estudia los productos científicos de manera similar a como el matemático estudia números, figuras geométricas y otras entidades matemáticas, como estructuras abstractas. En este sentido, la filosofía de la ciencia posee ciertas semejanzas de familia con la matemática pura.

Habíamos establecido que las teorías científicas son objetos culturales producidos por ciertas personas en situaciones socio-culturales dadas $y$ que, por esta razón, la filosofía de la ciencia forma parte de las ciencias de la cultura. Esto puede parecer que se halla en contradicción directa con el carácter abstracto, casi platónico, de la filosofía de la ciencia que acabamos de postular. Ahora bien, no creo que se dé una verdadera contradicción entre ambas caracterizaciones. Sería una concepción demasiado estrecha de las ciencias de la cultura creer que sólo pueden tratar de entidades localizadas espacio-temporalmente. Las teorías científicas son justamente un ejemplo de entidades culturales abstractas, no localizadas espacio-temporalmente. No hay, en efecto, ninguna razón para pensar que las humanidades están restringidas a priori al estudio de entidades concretas como pueden ser los hombres o las sociedades. Si estamos dispuestos a salirnos de un marco estrechamente empirista y nominalista de fundamentación de las ciencias de la cultura, podemos admitir fácilmente la posibilidad de que éstas estudien no sólo las realizaciones culturales concretas, los contextos sociales concretos en que se manifiestan y los individuos concretos que las producen, sino también las estructuras conceptuales generales subyacentes a ellas en definitiva, las formas culturales abstractas de las que esas realizaciones concretas son una instancia suya. Incluso podríamos formular nuestra tesis en un sentido más fuerte: yo me atrevería a afirmar que las ciencias de la cultura sólo podrán romper el estrecho marco que actualmente limita su progreso y que las hace aparecer como la hermana pobre ante las ciencias naturales, a partir del momento en que se decidan a pasar de manera sistemática al nivel de absiracción que justamente es característico de las ciencias naturales más avanzadas, en particular de la fisica matemática.

La física moderna dio el "Gran Salto Adelante", que le permitió ocupar el trono de Reina de las Ciencias, con Newton, es decir, cuando se atrevió a construir un marco conceptual abstracto que aparecía como independiente y hasta ajeno a las características concretas de los diversos sistemas físicos. Desde este nivel de suprema abstracción se descendió luego al estudio de las instancias físicas concretas, iluminadas ahora por una nueva luz, mucho más potente que todos los esquemas anteriores a Newton, la luz de las formas abstractas. En Newton el salto aún fue tímido y con vacilaciones, implícito, influido como estaba él por una metodología inductivista y empirista que le conducía a negar sobre el papel lo que él 
mismo estaba practicando de facto. En cualquier caso, a pesar de estas vacilaciones e inconsistencias metodológicas, el primer paso estaba ya dado, y toda la historia de la mecánica en el siglo XVIII no es sino la persecución sistemática del ideal de la máxima abstracción, persecución que culmina en la obra de Lagrange a fines de aquel siglo. Sucesivamente se rindieron al avance de la abstracción formal campos de fenómenos que parecían tan recalcitrantes a la conceptualización abstracta como son el calor, la electricidad o las reacciones químicas; la teoria del calor de Fourier, la electrodinámica de Maxwell y la termodinámica química de Gibbs son otros tantos hitos en una evolución conceptual que a primera vista parecía alejarnos cada vez más de la realidad concreta de nuestra experiencia humana, pero que al final resultó que nos hacía inteligibles y dominables fenómenos tan cotidianos como un relámpago o la fusión del hielo en el agua. En todos estos casos, naturalmente, se utilizaron métodos de formalización muy distintos de los de la mecánica; la mecánica fue un paradigma para otras disciplinas no en el sentido de que se tomaran prestadas de ella sus métodos formales, sino de que impulsó la búsqueda de herramientas adecuadas a cada campo, que cumplieran en último término el mismo cometido que se había alcanzado por vez primera en la mecánica.

Me he extendido un poco en el ejemplo de la génesis de la física teórica, no para proponer una burda asimilación de las ciencias humanas a las ciencias físicas, sino para hacer notar dos puntos. Primero, que el mejor camino para el dominio de la realidad más concreta pasa por la suprema abstracción formal; segundo, que fenómenos al parecer demasiado complejos para ser susceptibles de estudio sistemático pueden llegar a ser dominados conceptualmente si somos capaces de dar con las herramientas formales adecuadas al caso.

A veces se arguye que las ciencias de la cultura no pueden ni siquiera plantearse la posibilidad de alcanzar un nivel de abstracción semejante al de las ciencias físicas, debido a la extrema complejidad de su objeto de estudio. Pero, si se admite lo que acabamos de observar sobre la evolución de la física, se echará de ver que esta objeción carece de fuerza probatoria. También los fenómenos térmicos, eléctricos o químicos parecían a mucha gente hasta hace siglo y medio demasiado complejos en su diversidad concreta como para ser subsumibles bajo esquemas formales. Sin embargo, los denodados esfuerzos de unos cuantos hombres demostraron que esa actitud derrotista no estaba justificada. Claro que esto no fue tarea fácil. Al principio, los obstáculos parecían insuperables. Pero lo que hicieron esos hombres fue buscar detrás de la infinita variedad de manifestaciones de aquellos fenómenos las estructuras esenciales conceptualizables. No hay razón para pensar que lo mismo no se puede llevar a cabo en el caso de los objetos culturales. Es más, muchos científicos de la cultura han em- 
prendido ya este camino con éxitos notables desde hace un par de décadas. Musicólogos, críticos literarios y en particular lingüistas se dedican cada vez más sistemáticamente a indagar cuáles sean las estructuras abstractas que subyacen y hacen inteligibles los objetos concretos de su estudio, los cuales son sin duda objetos culturales.

Detengámonos un momento en el caso más sobresaliente, el de la linguística moderna. Ya Saussure, con su distinción entre langue y parole (es decir, entre lengua y habla), hizo ver a los linguiistas la posibilidad y necesidad de trascender las concreciones empíricas que constituyen el habla, con su infinita variedad de matices, para adentrarse en el campo de la lengua, constituida por formas abstractas generales. Por supuesto que Saussure admitía una conexión entre lengua y habla, y que las consideraciones teóricas sobre las estructuras formales de la lengua deben tener su traducción a ciertas regularidades empíricas detectadas en el habla; de lo contrario, el estudio sería una pura especulación sin fundamento en la realidad. Pero el núcleo del programa de Saussure era que el habla concreta sólo se podría hacer inteligible desde las estructuras formalizadas al nivel de la lengua. Estas últimas, por supuesto, no son entidades localizadas especialmente.

Esta tendencia de la lingǘstica hacia la búsqueda de un nivel de abstracción adecuado se ha hecho aún más notoria en las tres últimas décadas, sobre todo después de Chomsky, con el advenimiento de la lingüística generativo-transformacional. No en vano el punto de partida metodológico de esta teoría es una distinción análoga a la de Saussure: la distinción entre estructura superficial y estructura profunda de la lengua, hecha de manera aún más sistemática que en Saussure. La gramática que la lingüística generativo-transformacional trata de establecer equivale a la estructura profunda de la lengua y sólo indirectamente se revela en los modos concretos de hablar de la gente.

Hay además otro punto que merece ser notado respecto a esta disciplina, y es el que se refiere al uso sistemático y deliberado de ciertos instrumentos formales. Los notables avances de la lingüística generativotransformacional no habrían sido posibles si esta teoría no se hubiera decidido a aplicar una nueva herramienta matemática: la teoría de automatas. Según Chomsky y sus colaboradores, las estructuras profundas de la gramática de la lengua sólo pueden ser plasmadas adecuadamente en términos de la teoría formal de autómatas. Y lo curioso del caso es que esta teoria formal no ha sido tomada prestada de la física; la teoría de autómatas apenas tiene alguna aplicación en física. Al buscar los instrumentos formales adecuados, no se trata pues de imitar a la física en sus métodos específicos de conceptualización, sino en todo caso de adoptar la idea de buscar lo que necesitemos en cada caso para lograr la máxima precisión conceptual y la máxima potencia de sistematización. 
El ejemplo de la lingüística es especialmente relevante para nuestra discusión. Hay más de una afinidad entre el modo de proceder de la lingüística reciente y el que debería ser el de la filosofía de la ciencia. En efecto, ya el filósofo positivista Karl Pearson había dicho hace un siglo que el objeto de estudio de la filosofía de la ciencia debía ser la gramática de la ciencia. Si este término se entiende no en el sentido de la gramática escolar ordinaria, sino en el de las estructuras profundas de Chomsky, entonces podemos concordar plenamente con $\mathrm{K}$. Pearson. También la filosofía de la ciencia, en cuanto que es teorización de teorizaciones, debe romper la superficie de las teorías científicas en sus modos de manifestación concreta para adentrarse en las estructuras implícitas subyacentes que hacen inteligibles esas manifestaciones concretas. De lo contrario, nunca entenderemos lo que es esencial en la ciencia (y que probablemente escape a los propios científicos) y los filósofos no quedaríamos limitados al papel de divulgadores científicos, un papel que puede ser muy importante por otras razones, pero que ciertamente no es una tärea filosófica.

$\mathrm{Y}$ al igual que las diversas ramas de la física y de la lingüística moderna, también la filosofía de la ciencia debe adquirir o forjar sus propias armas de aprehensión conceptual. La filosofia de la ciencia puede y debe llegar a ser una disciplina tan rigurosa como la que más. Su ubicación dentro de las ciencias de la cultura, como ya hernos argüido, no es impedimento alguno para que emplee métodos formales de análisis. Pero como ya hemos advertido también la elección de los instrumentos formales no debe imitar ciegamente las elecciones hechas en otras disciplinas. Lo que se trata de asimilar es la idea general, no la ejemplificación característica en una disciplina particular. La herramienta formal que ha demostrado ser más adecuada y potente para la física es el cálculo diferencial; la que, al menos de momento, parece más apropiada para la linguíística es la teoría de autómatas. No obstante, la filosofía de la ciencia no tiene por qué adoptar ni la una ni la otra; es más, intuitivamente parece claro que ninguna de las dos le serviría, puesto que la formalización de los cambios físicos o de las regularidades del habla no puede proceder de la misma manera que la formalización de esos objetos culturales tan abstractos que llamamos «teorías científicas».

¿Cuál es el método formal adecuado a la tarea de la filosofía de la ciencia? Durante varias décadas, desde que se inició la filosofía de la ciencia como disciplina en sentido estricto, muchos autores, con Carnap a la cabeza, pensaron que la mejor herramienta para el análisis de las teorías científicas era la lógica, o aún más específicamente, la lógica de predicados de primer orden. Esta idea no era inverosímil si se tiene en cuenta que, desde principios de siglo, la lógica había prestado enormes servicios a la metamatemática, o sea, a las teorizaciones de segundo nivel sobre las teorías matemáticas. Quizás era natural pensar que la formalización lógica 
se podría trasladar, sin más, al estudio de las teorias empíricas. Pero la experiencia ha mostrado que ello no es así. La lógica no es la mejor herramienta para tratar las estructuras más interesantes que se presentan en las ciencias empíricas. No se trata de que la lógica sea completamente inútil. Sigue siendo un instrumento indispensable para iniciarse en el proceder analítico de la filosofía de la ciencia. Pero no podemos quedarnos ahí. Los aspectos más complejos de las ciencias empíricas rompen el marco estrecho que presupone la lógica. Es necesario complementarla con herramientas matemáticas más potentes. Por mi parte, me adscribo a una tendencia cada vez más fuerte en los últimos treinta años a usar la teoría de conjuntos y la teoria de modelos en este campo. Por supuesto, no hay ningún argumento definitivo a priori que demuestre que este enfoque sea el óptimo. En filosofía de la ciencia, lo mismo que en la propia ciencia o en cualquier otra actividad humana que aspire a hacer las cosas bien, hay que ir probando pacientemente diversos métodos hasta que encontremos el que parece más adecuado a nuestro objetivo. Nada se nos da regalado. Todo propósito intelectual serio requiere, como decía Hegel, "tomar sobre sí el esfuerzo del concepto». Un esfuerzo infatigable de esta clase es lo que necesitamos para desenterrar las estructuras esenciales de este fenómeno cultural tan pasmoso y difícil de apresar que llamamos ciencia. 